\title{
A Case Report of Pre-excited Atrial Fibrillation Encounters Ebstein's Anomaly: Is Amiodarone a Good Alternative Choice?
}

\author{
Hsiang-Wei Yang ${ }^{*}$ and Wai-Kin Wong
}

Department of Cardiology, Hsinchu Mackay Memorial Hospital, Hsinchu City, Taiwan

*Corresponding author: Dr. Hsiang-Wei Yang, Department of Cardiology, Hsinchu Mackay Memorial Hospital, No. 690 Section 2, Guangfu Road, Hsinchu City 300, Taiwan, Tel: 886-3-611-9595 ext. 2331, Fax: 886-3-611-1175

\section{Introduction}

This is a case report of a 39-year-old man who presented to the emergency room with atrial fibrillation $(\mathrm{AF})$, pre-excitation, and Ebstein's anomaly (EA). Currently, there was no suggestion of pharmacologic management if the patient declines surgical or catheter ablation therapy. A review of the literature concluded that amiodarone may be a suitable therapy for the pharmacological management of this patient.

\section{Case Description}

This 39-year-old man experienced occasional palpitations but on this occasion, the palpitations were worse with chest pressure during his morning sleep. He visited the emergency department with a pulse rate of 150 per minute, and electrocardiography showed a wide QRS-complex tachycardia in irregular beats (Figure 1) but laboratory tests showed only hypophosphatemia $(1.3 \mathrm{mg} / \mathrm{dL})$. Cardioversion was suggested for the developing ventricular tachycardia (VT) and ventricular fibrillation (VF) but he declined. Other suggested intravenous medications were unavailable, so he received a gentle amiodarone infusion which gradually recovered arrhythmia within a few hours. The recovered electrocardiography disclosed Wolff-Parkinson-White (WPW) syndrome. The thyroid function test, followed serum phosphate, and 24-hour electrocardiography were normal. The echocardiography revealed interventricular septal hypertrophy, right atrial and ventricular enlargement, and dislocation of the tricuspid valve toward the apex (Figure 1). With the combination of EA, WPW syndrome, and paroxysmal $\mathrm{AF}$, he refused cardiac ablation. After dosage adjustment over several months, the patient was managed with $200 \mathrm{mg}$ amiodarone daily.

\section{Discussion}

Pre-excited AF (AF with ventricular pre-excitation), supraventricular tachycardia with aberrant conduction, and ventricular tachycardia should be considered for irregular wide-complex tachycardia. The significant irregular RR interval with varying QRS morphology is characteristic of pre-excited AF, in which the electrical impulses via the AP (accessory pathway) exceed the AV nodal pathway. In this condition, intravenous amiodarone, adenosine, and other AV nodal blocking agents may lose the intrinsic inhibition of orthodromic conduction, accelerate the ventricular rate or contribute to VT or VF. According to current guidelines, prompt direct-current cardioversion is recommended for hemodynamically compromised patients, with the administration of intravenous Ibutilide or procainamide to stabilize the patient. Ibutilide and procainamide prolong the refractoriness of both pathways and Ibutilide helps to terminate AF [1]. However, these medications are not easily obtainable, and the intravenous infusion of amiodarone is regarded as a class III recommendation.

In Korea, flecainide was used to maintain sinus rhythm in an older patient with paroxysmal AF and

Citation: Hsiang-Wei Y, Wai-Kin W (2021) A Case Report of Pre-excited Atrial Fibrillation Encounters Ebstein's Anomaly: Is Amiodarone a Good Alternative Choice?. Int J Clin Cardiol 8:240. doi. org/10.23937/2378-2951/1410240

Accepted: October 21, 2021: Published: October 23, 2021

Copyright: ( 2021 Hsiang-Wei Y, et al. This is an open-access article distributed under the terms of the Creative Commons Attribution License, which permits unrestricted use, distribution, and reproduction in any medium, provided the original author and source are credited. 

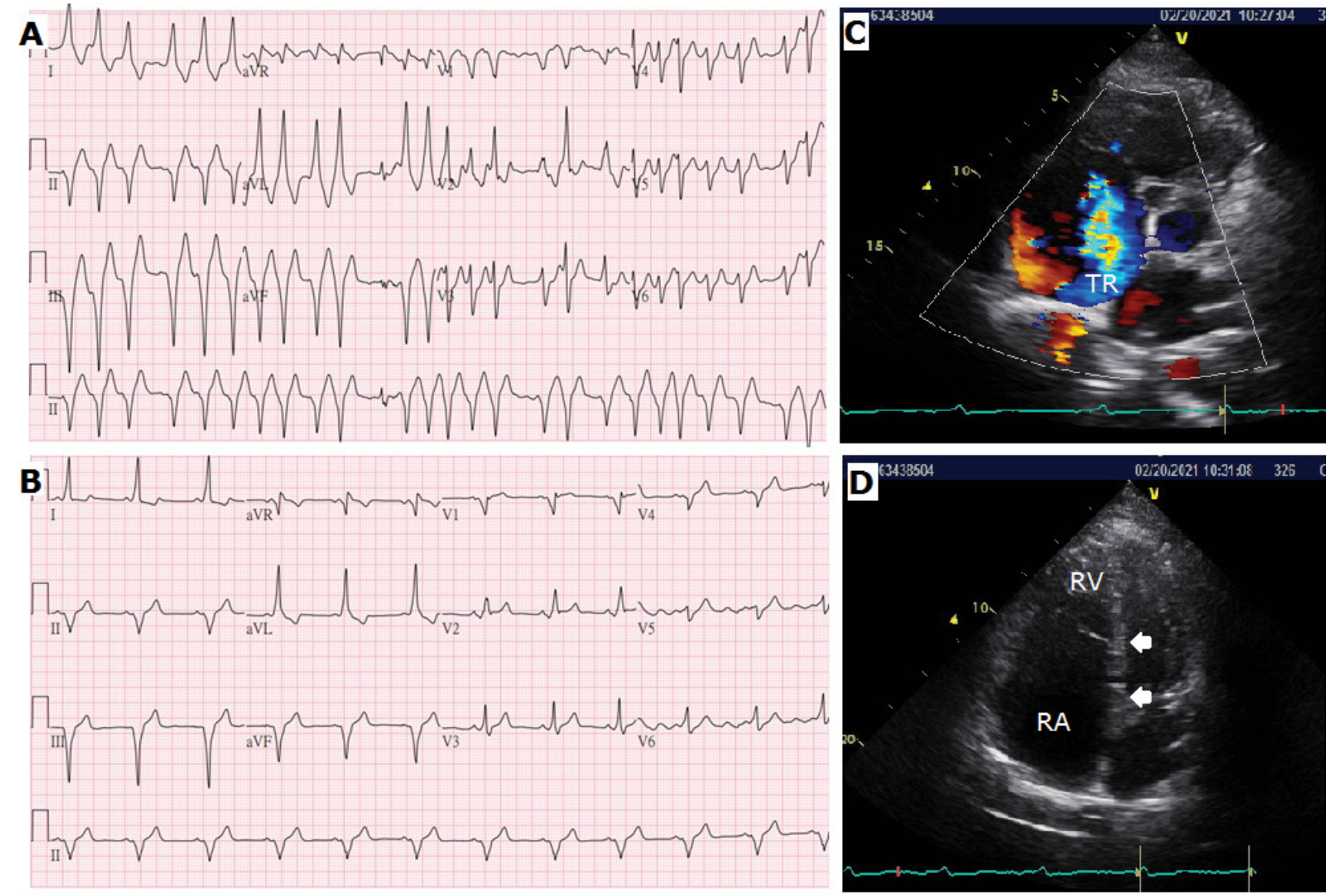

Figure 1: A) Electrocardiography initially showed a heart rate of 150 beats per minute, and the irregular interval without a predictable period indicated atrial fibrillation. There were wide QRS-complexes with varying QRS morphology, which were related to atrial fibrillation with different degrees of aberrant conduction; B) Electrocardiography after successful cardioversion showed a sinus rhythm of 68 beats per minute. The short PR interval $(<120 \mathrm{~ms})$ and a slurred sloping wave (delta wave) were the typical signs of Wolff-Parkinson-White syndrome. The dominant $\mathrm{S}$ wave in $\mathrm{V} 1$ indicated a right- sided accessory pathway; C) There was significant right atrial (RA) enlargement and moderate tricuspid regurgitation (TR) from the short-axis view of the echocardiograph. The estimated systolic pulmonary artery pressure was $35 \mathrm{mmHg}$; D) The four- chamber view showed displacement of $29 \mathrm{~mm}$ between the insertion site of the septal tricuspid leaflet (upper arrow) and the septal mitral valve (lower arrow). There was a large area of atrialization of the right ventricle (RV), resulting in a very small residual RV. There was no atrial septal defect found in the patient.

WPW. Flecainide maintains the sinus rate with little effect on the AV nodal refractory period in patients without structural heart disease. The concomitant administration of low dose bisoprolol made the delta wave disappear [2]. In a retrospective review of the administration of intravenous amiodarone for patients with pre-excited AF, most patients $(18 / 30,60 \%)$ achieved sinus rhythm without electrical cardioversion [3]. Intravenous amiodarone for patients with pre-excited $\mathrm{AF}$ is a treatment option if the guideline-suggested managements are unavailable or initially undesirable, but not recommended for patients with unstable hemodynamics, extremely high heart rate, multiple comorbidities, and younger patients. The rhythm may become VF at any time, even before the administration of intravenous amiodarone, hence a defibrillator should be readily available.

However, management becomes more complicated when the patient has concurrent EA. Patients with EA may develop cyanosis, exercise tolerance, heart failure, embolization, and arrhythmia. The arrhythmogenic atrialized right ventricle causes AV conduction delay and atrial tachyarrhythmia. The dislocated tricuspid valve elongates the tricuspid annulus, and makes the formation of the right-sided AP, especially on the lower half of the tricuspid annulus [4]. In fact, these patients have more complicated AP. Up to $50 \%$ of patients with EA have more AP, multiple AP, or board AP. Surgical or catheter intervention is suggested for symptomatic patients but these procedures are challenging, but it remains a highly effective and curative management [5]. Without intervention, adults with EA are more likely to die from sudden cardiac death rather than heart failure [6], hence pharmacological prevention of tachyarrhythmia is reasonable. Amiodarone is recommended for $A F$ with heart failure or structural heart disease as it maintains sinus rhythm, thus may be appropriate for patients with uncorrected paroxysmal $A F, W P W$, and $E A$.

\section{Conclusions}

In a patient with pre-excited $A F$, the recommended 
therapy is the administration of IV procainamide or Ibutilide, cardioversion, and catheter ablation of the accessory pathway. IV amiodarone might be an alternative therapy for pre-excited AF, but a defibrillator should be readily available. In a patient with paroxysmal AF, WPW and Ebstein's anomaly, oral amiodarone to maintain sinus rhythm might be reasonable.

\section{Conflict of Interests}

All the authors declare no conflict of interest.

\section{Sources of Support}

There were no sources of support.

\section{Statement of Equal Authors' Contribution}

All authors made equal contribution.

\section{References}

1. January CT, Wann LS, Alpert JS, Calkins H, Cigarroa JE, et al. (2014) 2014 AHA/ACC/HRS guideline for the management of patients with atrial fibrillation: Executive Summary. Circulation 130: 2071-2104.
2. Kwak JJ, Doh JH, Lee SY (2019) Effects of bisoprolol and flecainide in an elderly patient with paroxysmal supraventricular tachycardia and atrial fibrillation in WolffParkinson-White Syndrome. Int J Clin Cardiol 6: 138.

3. Ren J, Yang Y, Zhu J, Wu S, Wang J, et al. (2020) The use of intravenous amiodarone in patients with atrial fibrillation and Wolff-Parkinson-White syndrome. Pacing Clin Electrophysiol 44: 35-43.

4. Wei W, Zhan X, Xue Y, Fang X, Liao H, et al. (2014) Features of accessory pathways in adult Ebstein's anomaly. Europace 16: 1619-1625.

5. Orczykowski $M$, Derejko $P$, Bodalski $R$, Urbanek $P$, Zakrzewska-Koperska J, et al. (2017) Radiofrequency catheter ablation of accessory pathways in patients with Ebstein's anomaly: At 8 years of follow-up. Cardiol $\mathrm{J} \mathrm{24:}$ 1-8.

6. Eftekhari H, Osman F (2011) Wolff-Parkinson-White syndrome, pre-excited AF and Ebstein's anomaly: A case study. British Journal of Cardiac Nursing 6: 444-449. 